\title{
Aa. Vv., Relire "Le Rouge et le Noir", sous la direction de Xavier Bourdenet, Pierre Glaudes et François Vanoosthuyse
}

Michel Arrous

\section{OpenEdition}

Journals

Édition électronique

URL : http://journals.openedition.org/studifrancesi/1542

DOI : $10.4000 /$ studifrancesi. 1542

ISSN : 2421-5856

Éditeur

Rosenberg \& Sellier

Édition imprimée

Date de publication : 1 novembre 2014

Pagination : 613-615

ISSN : 0039-2944

\section{Référence électronique}

Michel Arrous, "Aa. Vv., Relire "Le Rouge et le Noir", sous la direction de Xavier Bourdenet, Pierre Glaudes et François Vanoosthuyse », Studi Francesi [En ligne], 174 (LVIII | III) | 2014, mis en ligne le 01 novembre 2014, consulté le 18 septembre 2020. URL : http://journals.openedition.org/studifrancesi/ 1542 ; DOI : https://doi.org/10.4000/studifrancesi.1542

Ce document a été généré automatiquement le 18 septembre 2020.

\section{cc) (†) $\odot$}

Studi Francesi è distribuita con Licenza Creative Commons Attribuzione - Non commerciale - Non opere derivate 4.0 Internazionale. 


\title{
Aa. Vv., Relire "Le Rouge et le Noir", sous la direction de Xavier Bourdenet, Pierre Glaudes et François Vanoosthuyse
}

\author{
Michel Arrous
}

\section{RÉFÉRENCE}

AA. VV., Relire "Le Rouge et le Noir", sous la direction de Xavier BOURDENET, Pierre GLAUDES et François VANoosthuYSE, Paris, Classiques Garnier, 2013, pp. 385.

1 À l'exception de celles de Pierre Glaudes et de Mariella Di Maio, et d'une traduction bienvenue d'une étude de Peter Brooks (PMLA, mai 1982) reprise sous le titre Le roman et la guillotine ou Pères et Fils dans "Le Rouge et le Noir" (dans Reading for the Plot, 1984, pp. 243-271), les contributions à ce volume ont été présentées lors du colloque organisé par le CPR 19 de la Sorbonne Nouvelle et le CRLF XIX ${ }^{e}$ de Paris-Sorbonne, le 7 décembre 2013. La plupart des nouveaux enjeux de la critique «rougiste» sont pris en compte dans quatre parties de cohérence parfois inégale: «Roman et politique»; «Logique, structure, sens»; «Les personnages et leur mise en scène»; «Langage, style, tonalité».

Yves ANSEL ("Quoiqu'il soit ultra et moi libéral...» "Le Rouge et le Noir", roman partisan, miroir déformant, pp. 15-32) rappelle qu'aux yeux des contemporains ce récit politique, "partial, satirique, polémique», était rien moins que fidèle à l'annonce de son épigraphe. Raillant non sans raisons les lectures textualistes qui font l'impasse sur la «Chronique de 1830», l'auteur peut affirmer qu'on a plus affaire à un roman partisan qu'à un «miroir sans parti pris». L'étude qui suit, "Le Rouge et le Noir" et la Révolution: les pathologies de la liberté (pp. 33-47), nuance la stylisation dont Stendhal serait coupable. S'interrogeant sur le statut de la Révolution dans le roman, Marie-Rose CORREDOR y montre que, même ternie, son aura fantasmatique n'en agit pas moins dans la société 
bloquée de la Restauration. Autre exemple de textualisation du discours politique avec «Les plaisirs de la campagne» (II, I), petit apologue satirique dans lequel l'histoire contemporaine est intégrée au récit romanesque par le biais de Julien, plus observateur qu'acteur, et de l'auteur encore une fois polémiste. Xavier BOURDENET revient sur le cotexte proliférant qui a nourri l'argumentation à l'œuvre dans tout le chapitre: la politisation envahissante et irrémédiable qui caractérise la modernité interdit tout refuge dans la nature ( $« S i$ vos personnages ne parlent pas de politique, ce ne sont plus de Français de 1830», pp. 49-67). Comme on ne peut réduire le roman à une satire de la monarchie restaurée, de l'aristocratie et du clergé, Pierre GLAUDEs propose de le lire comme une mise en question de la communauté livrée aux conflits idéologiques, qu'il s'agisse de la conception maistrienne de la communauté aussi bien que des mœurs libérales que le jacobinisme stendhalien n'épargne pas. Il y a néanmoins un mythe de la communauté heureuse quand, dans la prison, Julien et Mme de Rênal se dévouent l'un à l'autre ("Le Rouge et le Noir": malaise dans la communauté, pp. 69-97).

3 S'il est possible de repérer de nombreuses marques de dualité dans ce roman que caractérise la dialectique du contrepoint ou le conflit de forces opposées, Jacques-David EBGUY préfère en comprendre le sens et l'effet ("Un se divise en deux». "Le Rouge et le Noir", roman du vivre, pp. 101-120). La pratique du renversement de la causalité et de la conjonction des dissemblables crée un «effet d'ambiguïté généralisée» dont Stendhal ne cesse de jouer. Dans tout le roman, «une loi de la disjonction semble à l'œuvre» qui renvoie à l'ambiguïté fondamentale de l'époque. Dans «Chapitre ce me semble». "Le Rouge et le Noir" et ses soixante-quinze chapitres (pp. 121-141), Marie PARMENTIER se demande comment Stendhal traite la question de la segmentation en chapitres et quel est leur rôle. Si le dispositif choisi par Stendhal obéit à plusieurs motivations (changement dans la temporalité, dans l'espace, etc.), Le Rouge n'en est pas moins «un roman dont la structuration narrative ne se superpose généralement pas au découpage des chapitres»: c'est le cas, par exemple, du «séminaire», de la «Note secrète» ou lors des changements de lieu (le passage de la I ${ }^{\text {re }}$ à la $\mathrm{II}^{\mathrm{e}}$ partie en offre un exemple flagrant). Stendhal s'approprie la norme de son temps (la division en chapitres), sans pour autant respecter l'unité textuelle. Catherine maRietTe-CLOT revient sur l'article en forme de mise au point et d'analyse sociologique, à vrai dire un peu schématique, que Stendhal destinait à V. Salvagnoli pour qu'il rende compte de son roman (Le Projet d'article sur "Le Rouge et le Noir", pp. 143-157). Voulant se distinguer du roman historique (le modèle scottien répudié dans un article de 1830), comme du roman mélodramatique (l'in-12 « pour les femmes de chambre») ou du «roman des salons» (le roman sentimental illustré par Mme de Duras), Stendhal établit dans les modes de lecture et les lectorats une distinction principale Paris / province, que $\mathrm{C}$. Mariette-Clot reprend à son compte mais que les pratiques de l'époque infirment, si l'on en croit les catalogues des cabinets et salons de lecture. En revanche, les critiques que Stendhal adressait aux genres romanesques à la mode permettent de préciser sa conception du roman. Investigations inhabituelles en compagnie de Brigitte DIAZ (Verres verts et habits bleus. Le pouvoir des objets dans "Le Rouge et le Noir", pp. 159-178) qui démontre que Stendhal, contrairement à l'impression commune, s'intéresse aussi aux choses à travers sa sensibilité au détail. Bien que ténus, les usages qu'il en fait méritent d'être réévalués car dans Le Rouge l'objet est «assujetti à un dispositif d'ensemble dans lequel il prend sens».

On sait que le récit du «cœur mangé» apparaît dans deux rapides allusions des chapitres VIII et XXI, et, d'une manière développée, dans De l'Amour (chap. LII). Mariella 
DI MAIO, qui a déjà étudié cette thématique et les matériaux avant-textuels utilisés par Stendhal (Le Cour mangé. Histoire d'un thème littéraire du Moyen Âge au XIXe siècle), revient sur sa présence dans l'écriture stendhalienne, d'abord dans De l'Amour (La Provence au XII siècle), puis dans Le Rouge (Roman et Moyen Âge. Le cœur à Vergy, pp. 181-194). L'attention est attirée sur la transformation du thème dans le roman et une précise analyse des sources permet de s'interroger, d'une part, sur la reprise au second degré du thème, cette fois venu du théâtre et du mélodrame et non plus seulement des deux versions médiévales; d'autre part, sur sa signification dans le roman. Dans Le Rouge, roman sentimental quelque peu subversif, le sexe est voilé mais pas exclu, quoique l'auteur de L'Amant ait pu en dire. En guise de réplique, François VANOOSTHUYSE (La scène érotique du "Rouge". À propos d'un mot étrange de Marguerite Duras, pp. 195-212) propose de «voir ce qui est voilé, et de voir dans quelle mesure ça l'est». Ce roman d'amour est aussi un roman du sexe où la scénarisation de l'amour se fait sur le mode de la litote et de la métonymie, un roman dans lequel Éros est partout et partout lié aux enjeux sociopolitiques, d'où sa nature problématique. Avec le couple apparemment antithétique de Mme de Rênal et de Mathilde de La Mole, Stendhal aurait-il donné de la femme française de 1830 un panorama nuancé? Cécile MEYNARD analyse ce schéma binaire qui parut d'ailleurs déséquilibré à bien des lecteurs tant Mathilde peut sembler inférieure à Mme de Rênal (Construction et mise en scène des personnages féminins dans "Le Rouge et le Noir", pp. 213-232). Pourtant les points communs ne manquent pas! À ces deux figures centrales s'ajoutent, à l'arrière-plan, des personnages féminins secondaires avec des effets de dissemblance et de ressemblance, lesquels contribuent à la profondeur de la peinture de la femme de la Restauration. Philippe BERTHIER s'adonne à une subtile micro-lecture de l'épisode, à première vue anecdotique, de la Fête-Dieu à Besançon (I, XXVIII) (pp. 233-241). Étrange scène puisqu'elle «célèbre davantage le Corpus Dominae que le Corpus Domini». L'évanouissement de Mme de Rênal est décrypté sans pitié pour la victime d'une crise "obscurément voulue»: «Et pour cause: au cœur même du lieu saint, c'est à un contact érotique qu'elle a souhaité s'abandonner (levez-vous, orgasmes désirés!)». La contribution de Michel CROUZET («La guillotine, n'est-ce que ça?», pp. 273-304) est tout entière consacrée à la mort de Julien, à ce qu'elle représente et à ce qu'on doit comprendre de cette laideur qui est évoquée mais dont on ne dit rien. Plus que la mort, euphémisée, comptent la préparation à la mort et la mort vécue comme une action d'éclat. On se rappelle la phrase qui suit la méditation philosophique et religieuse: "Julien se sentait fort et résolu comme l'homme qui voit clair dans son âme ». C'est le cheminement vers cette résolution qui est analysé comme une «montée vers le courage», au terme de laquelle le héros est pleinement lui-même. Un héros qui parvient à se réhabiliter par l'estime qu'il a de lui-même, c'est-à-dire par la capacité à se surmonter.

Dans la dernière partie consacrée au thème du langage, Boris LYON-CAEN repère quatre pratiques d'écriture: la presse nourrit le roman, la pratique épistolaire est suggérée et représentée dans l'œuvre, la littérature omniprésente avec le "déjà écrit», et enfin l'œuvre devenant à elle-même sa propre référence ("Le Rouge et le Noir": le langage en représentation, pp. 307-321). Pour sa part, Éric BORDAS a choisi les réalités du style telles que Julien les découvre et apprend à les exploiter (Les leçons de stylistique appliquées de Julien Sorel, pp. 323-338). Julien s'initie d'abord aux parlures et aux sociolectes de la France de 1830 qui se partagent entre la province et Paris, entre l'aristocratie et la roture; mais le paramètre décisif, le plus subtil sans doute, c'est le ton dont Julien doit 
saisir toutes les nuances. La tradition «rougiste» a quelque peu négligé voire ignoré le rôle de l'humour pourtant bien présent et sous de nombreux aspects (comédie, ironie, satire, etc.). Ce sont ses effets que Christopher THOMPson relève dans le dernier volet du roman ( $L a$ fin du "Rouge et le Noir": fonctions des traits humoristiques, pp. 339-351) les effets perturbateurs qui rendent possibles plusieurs types de lectures. Un bel exemple en est donné avec l'imagination "gothique» de Mathilde parsemée de grains d'un «humour sombre» qui n'est pas sans rappeler Shakespeare. 